\title{
Two distance-regular graphs
}

\author{
Andries E. Brouwer • Dmitrii V. Pasechnik
}

Received: 11 June 2011 / Accepted: 5 December 2011 / Published online: 20 December 2011

(C) The Author(s) 2011. This article is published with open access at Springerlink.com

\begin{abstract}
We construct two families of distance-regular graphs, namely the subgraph of the dual polar graph of type $B_{3}(q)$ induced on the vertices far from a fixed point, and the subgraph of the dual polar graph of type $D_{4}(q)$ induced on the vertices far from a fixed edge. The latter is the extended bipartite double of the former.
\end{abstract}

Keywords Distance-regular graph · Dual polar graph · Extended bipartite double

\section{The extended bipartite double}

We shall use $\sim$ to indicate adjacency in a graph. For notation and definitions of concepts related to distance-regular graphs, see [3]. We repeat the definition of extended bipartite double.

The bipartite double of a graph $\Gamma$ with vertex set $X$ is the graph with vertex set $\left\{x^{+}, x^{-} \mid x \in X\right\}$ and adjacencies $x^{\delta} \sim y^{\epsilon}$ iff $\delta \epsilon=-1$ and $x \sim y$. The bipartite double of a graph $\Gamma$ is bipartite, and it is connected iff $\Gamma$ is connected and not bipartite. If $\Gamma$ has spectrum $\Phi$, then its bipartite double has spectrum $(-\Phi) \cup \Phi$. See also [3], Theorem 1.11.1.

The extended bipartite double of a graph $\Gamma$ with vertex set $X$ is the graph with vertex set $\left\{x^{+}, x^{-} \mid x \in X\right\}$, and the same adjacencies as the bipartite double, except that also $x^{-} \sim x^{+}$for all $x \in X$. The extended bipartite double of a graph $\Gamma$ is bipartite, and it is connected iff $\Gamma$ is connected. If $\Gamma$ has spectrum $\Phi$, then its extended bipartite double has spectrum $(-\Phi-1) \cup(\Phi+1)$. See also [3], Theorem 1.11.2.

\footnotetext{
A.E. Brouwer $(\bowtie)$

Dept. of Math., Techn. Univ. Eindhoven, P.O. Box 513, 5600MB Eindhoven, Netherlands e-mail: aeb@cwi.nl

D.V. Pasechnik

School of Physical and Mathematical Sciences, Nanyang Technological University, 21 Nanyang Link, Singapore 637371, Singapore

e-mail: dima@ntu.edu.sg
} 


\section{Far from an edge in the dual polar graph of type $D_{4}(q)$}

Let $V$ be a vector space of dimension 8 over a field $F$, provided with a nondegenerate quadratic form of maximal Witt index. The maximal totally isotropic subspaces of $V$ (of dimension 4) fall into two families $\mathcal{F}_{1}$ and $\mathcal{F}_{2}$, where the dimension of the intersection of two elements of the same family is even ( 4 or 2 or 0 ) and the dimension of the intersection of two elements of different families is odd (3 or 1).

The geometry of the totally isotropic subspaces of $V$, where $A \in \mathcal{F}_{1}$ and $B \in \mathcal{F}_{2}$ are incident when $\operatorname{dim} A \cap B=3$ and otherwise incidence is symmetrized inclusion, is known as the geometry $D_{4}(F)$. The bipartite incidence graph on the maximal totally isotropic subspaces is known as the dual polar graph of type $D_{4}(F)$.

Below we take $F=\mathbf{F}_{q}$, the finite field with $q$ elements, so that graph and geometry are finite. We shall use projective terminology, so that 1-spaces, 2-spaces and 3-spaces are called points, lines and planes. Two subspaces are called disjoint when they have no point in common, i.e., when the intersection has dimension 0 .

Proposition 2.1 Let $\Gamma$ be the dual polar graph of type $D_{4}\left(\mathbf{F}_{q}\right)$. Fix elements $A_{0} \in$ $\mathcal{F}_{1}$ and $B_{0} \in \mathcal{F}_{2}$ with $A_{0} \sim B_{0}$. Let $\Delta$ be the subgraph of $\Gamma$ induced on the set of vertices disjoint from $A_{0}$ or $B_{0}$. Then $\Delta$ is distance-regular with intersection array $\left\{q^{3}, q^{3}-1, q^{3}-q, q^{3}-q^{2}+1 ; 1, q, q^{2}-1, q^{3}\right\}$.

The distance distribution diagram is

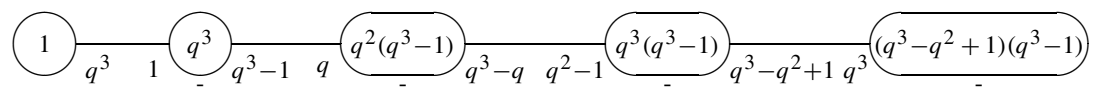

Proof There are $q^{6}$ elements $A \in \mathcal{F}_{1}$ disjoint from $A_{0}$ and the same number of $B \in \mathcal{F}_{2}$ disjoint from $B_{0}$, so that $\Delta$ has $2 q^{6}$ vertices.

Given $A \in \mathcal{F}_{1}$, there are $q^{3}+q^{2}+q+1$ elements $B \in \mathcal{F}_{2}$ incident to it. Of these, $q^{2}+q+1$ contain the point $A \cap B_{0}$ and hence are not vertices of $\Delta$. So, $\Delta$ has valency $q^{3}$.

Two vertices $A, A^{\prime} \in \mathcal{F}_{1}$ have distance 2 in $\Delta$ if and only if they meet in a line, and the line $L=A \cap A^{\prime}$ is disjoint from $B_{0}$. If this is the case, then $L$ is in $q+1$ elements $B \in \mathcal{F}_{2}$, one of which meets $B_{0}$, so that $A$ and $A^{\prime}$ have $c_{2}=q$ common neighbours in $\Delta$.

Given vertices $A \in \mathcal{F}_{1}$ and $B \in \mathcal{F}_{2}$ that are nonadjacent, i.e., that meet in a single point $P$, the neighbours $A^{\prime}$ of $B$ at distance 2 to $A$ in $\Delta$ correspond to the lines $L$ on $P$ in $A$ disjoint from $B_{0}$ and nonorthogonal to the point $A_{0} \cap B$. There are $q^{2}+q+1$ lines $L$ on $P$ in $A, q+1$ of which are orthogonal to the point $A_{0} \cap B$, and one further of which meets $B_{0}$. (Note that the points $A_{0} \cap B$ and $A \cap B_{0}$ are nonorthogonal since neither point is in the plane $A_{0} \cap B_{0}$ and $V$ does not contain totally isotropic 5-spaces.) It follows that $c_{3}=q^{2}-1$, and also that $\Delta$ has diameter 4 , and is distance-regular.

The geometry induced by the incidence relation of $D_{4}(F)$ on the vertices of $\Delta$, together with the points and lines contained in the planes disjoint from $A_{0} \cup B_{0}$, has Buekenhout-Tits diagram (cf. [4]) 


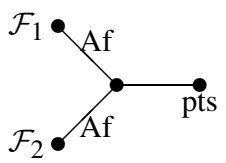

that is, the residue of an object $A \in \mathcal{F}_{1}$ is an affine 3-space, where the objects incident to $A$ in $\mathcal{F}_{2}$ play the rôle of points. Similar things hold more generally for $D_{n}(F)$ with arbitrary $n$, and even more generally for all diagrams of spherical type. See also [1], Theorem 6.1.

Let $P$ be a nonsingular point, and let $\phi$ be the reflection in the hyperplane $H=P^{\perp}$. Then $\phi$ is an element of order two of the orthogonal group that fixes $H$ pointwise, and consequently interchanges $\mathcal{F}_{1}$ and $\mathcal{F}_{2}$. For each $A \in \mathcal{F}_{1}$ we have $\phi(A) \sim A$. The quotient $\Gamma / \phi$ is the dual polar graph of type $B_{3}(q)$, and we see that more generally the dual polar graph of type $D_{m+1}(q)$ is the extended bipartite double of the dual polar graph of type $B_{m}(q)$. The quotient $\Delta / \phi$ is a new distance-regular graph discussed in the next section. It is the subgraph consisting of the vertices at maximal distance from a given point in the dual polar graph of type $B_{3}(q)$. For even $q$ we have $B_{3}(q)=C_{3}(q)$, and it follows that the symmetric bilinear forms graph on $\mathbf{F}_{q}^{3}$ is distance-regular, see [3] Proposition 9.5.10 and the diagram there on p. 286.

\section{Far from a point in the dual polar graph of type $B_{3}(q)$}

First a very explicit version of the graph of this section.

Proposition 3.1 (i) Let $W$ be a vector space of dimension 3 over the field $\mathbf{F}_{q}$, provided with an outer product $\times$. Let $Z$ be the graph with vertex set $W \times W$ where $\left(u, u^{\prime}\right) \sim\left(v, v^{\prime}\right)$ if and only if $\left(u, u^{\prime}\right) \neq\left(v, v^{\prime}\right)$ and $u \times v+u^{\prime}-v^{\prime}=0$. Then $Z$ is distance-regular of diameter 3 on $q^{6}$ vertices. It has intersection array $\left\{q^{3}-1, q^{3}-q, q^{3}-q^{2}+1 ; 1, q, q^{2}-1\right\}$ and eigenvalues $q^{3}-1, q^{2}-1,-1,-q^{2}-1$ with multiplicities $1, \frac{1}{2} q(q+1)\left(q^{3}-1\right),\left(q^{3}-q^{2}+1\right)\left(q^{3}-1\right), \frac{1}{2} q(q-1)\left(q^{3}-1\right)$, respectively.

(ii) The extended bipartite double $\hat{Z}$ of $Z$ is distance-regular with intersection array $\left\{q^{3}, q^{3}-1, q^{3}-q, q^{3}-q^{2}+1 ; 1, q, q^{2}-1, q^{3}\right\}$ and eigenvalues $\pm q^{3}, \pm q^{2}$, 0 with multiplicities $1, q^{2}\left(q^{3}-1\right), 2\left(q^{3}-q^{2}+1\right)\left(q^{3}-1\right)$, respectively.

(iii) The distance-1-or-2 graph $Z_{1} \cup Z_{2}$ of $Z$, which is the halved graph of $\hat{Z}$, is strongly regular with parameters $(v, k, \lambda, \mu)=\left(q^{6}, q^{2}\left(q^{3}-1\right), q^{2}\left(q^{2}+q-\right.\right.$ 3), $\left.q^{2}\left(q^{2}-1\right)\right)$.

The distance distribution diagram of $Z$ is

$$
\left.(1)_{q^{3}-1} \int_{q-2}^{q^{3}-1}\right)_{q^{3}-q} \frac{\left(q^{2}-1\right)\left(q^{3}-1\right)}{q^{2}-q-2} q^{3}-q^{2}+1 \quad q^{2}-1 \frac{\left(q^{3}-q^{2}+1\right)\left(q^{3}-1\right)}{q^{3}-q^{2}}
$$

Proof Note that the adjacency relation is symmetric, so that $Z$ is an undirected graph. The computation of the parameters is completely straightforward. Clearly, $Z$ has $q^{6}$ vertices. For $a, b \in W$ the maps $\left(u, u^{\prime}\right) \mapsto\left(u+a, u^{\prime}+(a \times u)+b\right)$ are automorphisms of $Z$, so $\operatorname{Aut}(Z)$ is vertex-transitive. 
The $q^{3}-1$ neighbours of $(0,0)$ are the vertices $(v, 0)$ with $v \neq 0$. The common neighbours of $(0,0)$ and $(v, 0)$ are the vertices $(c v, 0)$ for $c \in \mathbf{F}_{q}, c \neq 0,1$. Hence $a_{1}=q-2$.

The $\left(q^{3}-1\right)\left(q^{2}-1\right)$ vertices at distance 2 from $(0,0)$ are the vertices $\left(u, u^{\prime}\right)$ with $u, u^{\prime} \neq 0$ and $u^{\prime} \perp u{ }^{1}$ The common neighbours of $(0,0)$ and $\left(u, u^{\prime}\right)$ are the $(v, 0)$ with $v \times u=u^{\prime}$, and together with $(v, 0)$ also $(v+c u, 0)$ is a common neighbour, so $c_{2}=q$. Vertices $\left(u, u^{\prime}\right)$ and $\left(v, v^{\prime}\right)$, both at distance 2 from $(0,0)$, are adjacent when $0 \neq v \perp u^{\prime}$ and $v \neq u$ and $v \times u \neq u^{\prime}$ and $v^{\prime}=u \times v+u^{\prime}$, so that $a_{2}=q^{2}-q-2$.

The remaining $\left(q^{3}-1\right)\left(q^{3}-q^{2}+1\right)$ vertices have distance 3 to $(0,0)$. They are the $\left(w, w^{\prime}\right)$ with $w \not \perp w^{\prime}$ or $w=0 \neq w^{\prime}$. The neighbours $\left(u, u^{\prime}\right)$ of $\left(w, w^{\prime}\right)$ that lie at distance 2 to $(0,0)$ satisfy $0 \neq u \perp w^{\prime}$ and $(0 \neq) u^{\prime}=w \times u+w^{\prime}$, so that $c_{3}=q^{2}-1$. This shows that $Z$ is distance-regular with the claimed parameters. The spectrum follows.

The fact that the extended bipartite double is distance-regular, and has the stated intersection array, follows from [3], Theorem 1.11.2(vi).

The fact that $Z_{3}$ is strongly regular follows from [3], Proposition 4.2.17(ii) (which says that this happens when $Z$ has eigenvalue -1 ).

For $q=2$, the graphs here are (i) the folded 7-cube, (ii) the folded 8-cube, (iii) the halved folded 8-cube. All are distance-transitive. For $q>2$ these graphs are not distance-transitive.

When $q$ is a power of two, the graphs $\hat{Z}$ have the same parameters as certain Kasami graphs, but for $q>2$ these are nonisomorphic.

Next, a more geometric description of this graph.

Let $H$ be a vector space of dimension 7 over the field $\mathbf{F}_{q}$, provided with a nondegenerate quadratic form. Let $\Gamma$ be the graph of which the vertices are the maximal totally isotropic subspaces of $H$ (of dimension 3), where two vertices are adjacent when their intersection has dimension 2. This graph is known as the dual polar graph of type $B_{3}(q)$. It is distance-regular with intersection array $\left\{q\left(q^{2}+q+1\right), q^{2}(q+1), q^{3} ; 1, q+1, q^{2}+q+1\right\}$. (See [3], §9.4.)

Proposition 3.2 Let $\Gamma$ be the dual polar graph of type $B_{3}(q)$. Fix a vertex $\pi_{0}$ of $\Gamma$, and let $\Delta$ be the subgraph of $\Gamma$ induced on the collection of vertices disjoint from $\pi_{0}$. Then $\Delta$ is isomorphic to the graph $Z$ of Proposition 3.1. Its extended bipartite double $\hat{\Delta}$ (or $\hat{Z})$ is isomorphic to the graph of Proposition 2.1 .

Proof Let $V$ be a vector space of dimension 8 over $\mathbf{F}_{q}$ (with basis $\left\{e_{1}, \ldots, e_{8}\right\}$ ), provided with the nondegenerate quadratic form $Q(x)=x_{1} x_{5}+x_{2} x_{6}+x_{3} x_{7}+x_{4} x_{8}$. The point $P=(0,0,0,1,0,0,0,-1)$ is nonisotropic, and $P^{\perp}$ is the hyperplane $H$ defined by $x_{4}=x_{8}$. Restricted to $H$ the quadratic form becomes $Q(x)=x_{1} x_{5}+$ $x_{2} x_{6}+x_{3} x_{7}+x_{4}^{2}$.

The $D_{4}$-geometry on $V$ has disjoint maximal totally isotropic subspaces $E=$ $\left\langle e_{1}, e_{2}, e_{3}, e_{4}\right\rangle$ and $F=\left\langle e_{5}, e_{6}, e_{7}, e_{8}\right\rangle$. Fix $E$ and consider the collection of all maximal totally isotropic subspaces disjoint from $E$. This is precisely the collection of

${ }^{1}$ With orthogonality relation compatible with $\times$, so that $u \perp(u \times v)$ for all $u, v$. 
images $F_{A}$ of $F$ under matrices $\left(\begin{array}{ll}I & A \\ 0 & I\end{array}\right)$, where $A$ is alternating with zero diagonal (cf. [3], Proposition 9.5.1(i)). Hence, we can label the $q^{6}$ vertices $F_{A} \cap H$ of $\Delta$ with the $q^{6}$ matrices $A$.

Two vertices are adjacent when they have a line in common, that is, when they are the intersections with $H$ of maximal totally isotropic subspaces in $V$, disjoint from $E$, that meet in a line contained in $H$. Let

$$
A=\left(\begin{array}{cccc}
0 & a & b & c \\
-a & 0 & d & e \\
-b & -d & 0 & f \\
-c & -e & -f & 0
\end{array}\right)
$$

Then $\operatorname{det} A=(a f-b e+c d)^{2}$, and if $\operatorname{det} A=0$ but $A \neq 0$, then $\operatorname{ker} A$ has $\operatorname{dimension}$ 2 , and is spanned by the four vectors $(0, f,-e, d)^{\top},(-f, 0, c,-b)^{\top},(e,-c, 0, a)^{\top}$, $(-d, b,-a, 0)^{\top}$. Writing the condition that matrices $A$ and $A^{\prime}$ belong to adjacent vertices we find the description of Proposition 3.1 if we take $u=(c, e, f)$ and $u^{\prime}=$ $(-d, b,-a)$.

\section{History}

In 1991 the second author constructed the graphs from Sect. 2 and the first author those from Sect. 3. Both were mentioned on the web page [2], but not published thus far. These graphs have been called the Pasechnik graphs and the Brouwer-Pasechnik graphs, respectively, by on-line servers.

Open Access This article is distributed under the terms of the Creative Commons Attribution Noncommercial License which permits any noncommercial use, distribution, and reproduction in any medium, provided the original author(s) and source are credited.

\section{References}

1. Blok, R.J., Brouwer, A.E.: The geometry far from a residue. In: di Martino, L., Kantor, W.M., Lunardon, G., Pasini, A., Tamburini, M.C. (eds.) Groups and Geometries, pp. 29-38. Birkhäuser, Basel (1998)

2. Brouwer, A.E.: Additions and corrections to [3]. http://www.win.tue.nl/ aeb/drg/index.html

3. Brouwer, A.E., Cohen, A.M., Neumaier, A.: Distance-Regular Graphs. Springer, Heidelberg (1989)

4. Pasini, A.: Diagram Geometries. Oxford University Press, Oxford (1994) 